\title{
To Study the Vapour Liquid Equilibrium Data for Cellosolve Acetate and Toluene
}

\author{
VLE of Cellosolve and Toluene System
}

\author{
Uday Chavda, Shahnawaz Hala, Dhyey Savaliya, Milan Kathiriya \\ Chemical Engineering Department \\ Om Engineering College, Junagadh 362310 \\ Gujarat Technological University Ahmedabad, Gujarat, India
}

\begin{abstract}
Isobaric vapour-liquid equilibrium data for pure components as well as binary mixtures of Cellosolve Acetate-Toluene was generated using a modified ebulliometer. Measurements are reported for four different pressures in the range of (61 to 101) $\mathrm{kPa}$. Pure component vapor pressures were correlated using the Antoine correlation and compared with the literature data. Antoine constants are good match with literature and predicted data.
\end{abstract}

Keywords - Cellosolve Acetate; Toluene; VLE; Vapour-liquid

\section{INTRODUCTION}

\section{PHASE EQUILIBRIA (VAPOUR LIQUID EQUILIBRIUM):}

Distillation occupies a very important position in chemical engineering. Distillation and chemical reactors represent the backbone of what distinguishes chemical engineering from other engineering disciplines. Operations involving heat transfer and fluid mechanics are common to several disciplines. But distillation is uniquely under the purview of chemical engineers.

The basis of distillation is phase equilibrium, specifically, vapor-liquid (phase) equilibrium (VLE) and in some cases vapor-liquid-liquid (phase) equilibrium (VLLE). Distillation can effect a separation among chemical components only if the compositions of the vapor and liquid phases that are in phase equilibrium with each other are different. A reasonable understanding of VLE is essential for the analysis, design, and control of distillation columns.

Vapour liquid equilibrium (VLE) is a condition in which a liquid and vapour phase are in equilibrium with each other, a condition or state where the rate of evaporation equals the rate of condensation on molecular level such that there is no net vapour-liquid interconversion. A substance at vapour liquid equilibrium is generally referred to as a saturated fluid. For a pure chemical substance this implies that it is at its boiling point. Such VLE information is useful in designing columns for distillation, especially fractional distillation.

\section{EXPERIMENTALLY INVESTIGATED EQUILIBRIUM IN BINARY SYSTEM (CELLOSOLVE ACETATE- TOLUENE):}

VLE data on binary mixture of organic compounds are of significant importance for the design of numerous Industrial chemical processes or for the purpose of environmental protection. In the plants of various branches of Industry, where organic solvents are used, spend mixtures from a flow of toxic liquid wastes whose discharge in to the environmental is unacceptable. This makes necessary the development of technologies for recovering the starting solvents from the wastes and recycling them. For which phase equilibrium information is necessary. VLE studies of those systems are the goal of the present study. Following Systems have been studied,

2-Ethoxyethanol (Cellosolve) Acetate- Pure component

Toluene-Pure component

2-Ethoxyethanol (Cellosolve) Acetate-Toluene-Binary System

This binary system finds application in Esterification Reaction and Reactive distillation. The Vapour- Liquid Equilibrium (VLE) of this system is difficult to model especially due to High Boiling point and no data is available in the literature on this aspect to the best of my knowledge. Experiment P-T-x data of 2-Ethoxyethanol (Cellosolve) Acetate -Toluene system are generated for eight different set of composition at five different pressure for each set of Mixture and This Experimental data are used to regress Activity coefficient model parameters which are further used to generate P-T-x-y data. Results are compared with $\mathrm{G}^{\mathrm{E}}$ based Models (Margules 2-sufffix, Margules 3-suffix, NRTL, Vanlaar, Wilson Model).

\section{$G^{E}$ BASED MODELING}

Isobaric VLE data for the binary mixture of 2-Ethanolethanol (Cellosolve) Acetate - Toluene have been generated. The experimental data were correlated by using $\mathrm{G}^{\mathrm{E}}$ based Models (Margules 2-sufffix, Margules 3-suffix, NRTL, Vanlaar, Wilson Model).

Two vapour pressure models are used for the given system. For Cellosolve Acetate and for Toluene Antoine equation is used, whose constants are fitted to experimental Vapour pressure. By using regressed parameter BUBBLE $\mathrm{T}$ is found out.

Activity coefficient Model parameters are regressed using experimental P-T-x data. Then experimental binary P-T-x data modelled to find Vapour phase composition using regressed parameters and compared the vapour phase composition and temperature with BUBBLE T calculated data. 


\section{CHEMICALS}

\begin{tabular}{|c|c|c|c|}
\hline & M.W & B.P ${ }^{\circ} \mathbf{C}$ & Purity \% \\
\hline $\begin{array}{c}\text { Cellosolve (AR) } \\
\text { (2-Ethoxyethanol) } \\
\text { Acetate }\end{array}$ & & 156.2 & 99.0 \\
\hline Toluene & 132.16 & & \\
\hline IPA (Iso-propyl alcohol) & 92.14 & 110 & 99.0 \\
\hline
\end{tabular}

\section{VLE DATA GENERATION}

\section{PURE COMPONENT:}

\section{Component Name: CELLOSOLVE ACETATE (AR)}

Molecular Weight

Boiling Point

Purity

132.16

$: 156$

: $99 \%$

Density@20 ${ }^{\circ} \mathrm{C}$

0.9

Volume In

: $55 \mathrm{~m}$

Volume Out

$: 51 \mathrm{ml}$

\begin{tabular}{|c|c|}
\hline $\mathbf{P ~} \mathbf{~ m m H g}$ & $\mathbf{T}^{\circ} \mathbf{C}$ \\
\hline 759.21 & 156 \\
\hline 657.046 & 150.8 \\
\hline 553.96 & 143.8 \\
\hline 462.104 & 139.6 \\
\hline
\end{tabular}

Average Time for equilibrium: $60 \mathrm{~min}$

Vaporizing rate $\quad: 165 \mathrm{drops} / \mathrm{min}$

\section{Component Name: Toluene}

Molecular Weight $\quad: 92.14$

Boiling Point $\quad: 110.4$

Purity : $99 \%$

Density@ $20{ }^{\circ} \mathrm{C} \quad: 0.865$

Volume In $\quad: 55 \mathrm{ml}$

Volume Out $\quad: 50 \mathrm{ml}$

\begin{tabular}{|c|c|}
\hline $\mathbf{P} \mathbf{~ m m H g}$ & $\mathbf{T}^{\circ} \mathbf{C}$ \\
\hline $\mathbf{7 6 3 . 8 6 1}$ & 111 \\
\hline $\mathbf{6 5 8 . 7 3 2}$ & 105.2 \\
\hline $\mathbf{5 4 7 . 0 8 9}$ & 100.2 \\
\hline $\mathbf{4 6 4 . 9 3 1}$ & 94.8 \\
\hline
\end{tabular}

Average Time for equilibrium: $60 \mathrm{~min}$

Vaporizing rate $\quad: 165$ drops/min

\section{PURE COPMONENT MODELING}

\section{GENERAL MODELS:}

Vapour pressure is calculated by generally used models which are described here.

a) VAPOUR PRESSURE:

Antoine Equation:

$$
\operatorname{Ln} \mathrm{P}=\mathrm{A}-\left(\frac{B}{T+C}\right)
$$

Where: $\quad \mathrm{P}=$ Vapour pressure $\mathrm{KPa}$

$\mathrm{T}=$ Temperature in $\mathrm{K}$

A, B, C = Antoine Constants

Cellosolve Acetate and Toluene Vapour Pressure is found by the Antoine Equation Literature Antoine Constant

Table : Cellosolve Acetate and Toluene Literature Antoine Constant

\begin{tabular}{|c|c|c|c|}
\hline Component & A & B & C \\
\hline Cellosolve Acetate (ref_2) & 11.258 & 1620.024 & -185.83 \\
\hline Toluene (ref_1) & 14.0098 & 3100.01 & -53.36 \\
\hline
\end{tabular}

\section{MIXTURE MODELING:}

\section{a) GENERAL EQUILIBRIUM MODEL:}

$$
\begin{aligned}
& \text { Yi P } \emptyset_{i}=X_{i} \Upsilon_{i} P_{i}^{\text {sat }} \quad \text { for } \mathrm{i}=1,2 \ldots . \mathrm{N} \\
& \emptyset_{\mathrm{i}}=\frac{\text { di }^{\infty}}{\text { di sat }} \exp \frac{- \text { Vil }(p-\text { Pisat })}{R T} \\
& \mathrm{P}=\mathrm{P}^{\mathrm{T}} \mathrm{Z}_{\mathrm{i}}
\end{aligned}
$$

Where:

$$
\begin{array}{ll}
\mathrm{Yi}, \mathrm{Xi} & \text { Vapour and liquid mole fraction } \\
\mathrm{P}_{\text {sat }} & =\text { Saturated pressure } \\
\mathrm{P} & =\text { Corrected total pressure } \\
\mathrm{P}^{\mathrm{T}} & =\text { Total pressure } \\
\mathrm{R} & =\text { Universal gas constant } \\
\mathrm{Y}_{\mathrm{i}} & =\text { Activity Coefficient }
\end{array}
$$

\section{b) G $^{\mathrm{E}}$ BASED MODELING:}

Isobaric VLE data for the binary mixture of 2Ethoxyethanol (Cellosolve) - Toluene have been generated. The experimental data were correlated by using $\mathrm{G}^{\mathrm{E}}$ based models (Margules 2- suffix, Margules 3- suffix, Van laar, Wilson, and NRTL equations.) The experimental data were correlated by using the following $\mathrm{G}^{\mathrm{E}}$ based models:

- $\quad$ Margules 2- suffix model

- Margules 3- suffix model

- Van Laar model

- NRTL model

- Uniquac model

Vapour Pressure was calculated using the Antoine Equation. Parameters were regressed using the above mentioned models for all the binary mixture data and using them, BUBBL $\mathrm{T}$ or the bubble temperature was estimated.

Experimental P-T-x data are modelled to find Vapour phase composition using $\mathrm{G}^{\mathrm{E}}$ based models with regressed parameters. Activity coefficient model parameters are regressed using experimental P-T-x data. Then experimental $\mathrm{P}-\mathrm{T}-\mathrm{x}$ are modelled to find vapour phase composition and the comparison of the vapor phase composition and temperature with the calculated BUBBLE $\mathrm{T}$ data was made.

\section{GC PARAMETERS}

\begin{tabular}{|c|c|}
\hline GC PARAMETERS & CONDITIONS \\
\hline Column & Packed \\
\hline Range & $0-0$ \\
\hline Injection Port Temperature & $200{ }^{\circ} \mathrm{C}$ \\
\hline Detector Temperature & $210{ }^{\circ} \mathrm{C}$ \\
\hline Oven Temperature & $180{ }^{\circ} \mathrm{C}$ \\
\hline Carrier Gas (N2) Pressure & $0.8 \mathrm{bar}$ \\
\hline FID & $(+\mathrm{Ve})$ \\
\hline Injection Quantity & $0.1 \mu$ liter \\
\hline
\end{tabular}




\section{RESULT AND DISCUSSION}

Pure component and binary VLE data for Toluene Cellosolve Acetate system have been generated using a differential Ebulliometer with a provision of drop counter. The parameters of the Antoine equation for pure components have been regressed from the experimental data. Similarly, the parameters of various $\mathrm{G}^{\mathrm{E}}$ based models have been determined by regression using BUBL $\mathrm{T}$ calculations.

Experimental P-T-x data generated in this work are reported in chapter 3, the above system is modelled using various combinations of activity coefficient models and Table 5.1 shows pure component $\mathrm{P}-\mathrm{T}$ data. Table 5.8 shows regressed parameters of Activity coefficient models. Parameters are regressed at experimental P-T-x data generated in this work. The compositions reported are in terms of mole fraction with components ' 1 ' and ' 2 ' being Toluene and Cellosolve acetate respectively. Activity coefficient model parameters of Margules 2-suffix, Margules 3-suffix, Vanlaar, NRTL, Wilson parameters are regressed at experimental P-T-x data generated. BUBBLE $\mathrm{T}$ calculations performed by $\mathrm{G}^{\mathrm{E}}$ based models are reported in Table 5.4, 5.5, 5.6 and 5.7 and plotted in figure 5.4, 5.5, 5.6 and 5.7, \%ADD of experimental and model predicted BUBBLE T is calculated and tabulated in Tabls.

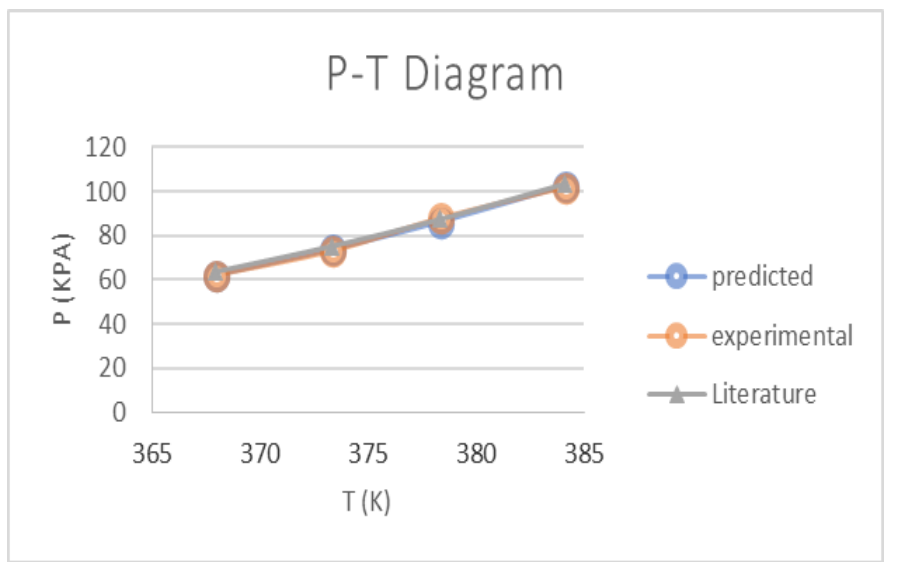

P-T diagrams for Predicted, experimental and literature values for Toluene.

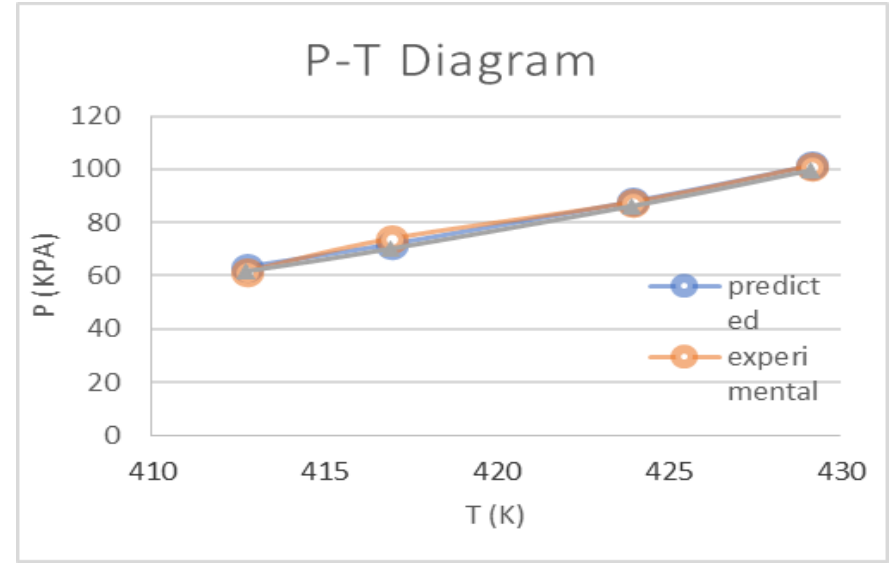

P-T diagrams for Predicted, experimental and literature values for Cellosolve Acetate.

\begin{tabular}{|l|l|l|l|l|l|}
\hline P KPa & $\mathbf{6 1 . 3 2}$ & $\mathbf{7 4 . 6 6}$ & $\mathbf{8 7 . 9 9}$ & $\mathbf{1 0 1 . 3 2 5}$ & Toluene \\
\hline & 408.0 & 412.4 & 418.2 & 420.4 & 5 \\
\hline & 399.0 & 406.0 & 410.2 & 415.4 & 15 \\
\hline & 393.6 & 398.0 & 402.2 & 406.8 & 26 \\
\hline \multirow{4}{*}{ T in K } & 381.2 & 387.0 & 393.4 & 400.0 & 38 \\
\hline & 382.6 & 388.4 & 393.4 & 397.4 & 46 \\
\hline & 378.0 & 383.6 & 389.0 & 393.6 & 55 \\
\hline & 374.6 & 378.4 & 383.2 & 390.6 & 69 \\
\hline & 372.0 & 377.8 & 383.4 & 388.4 & 76 \\
\hline 369.4 & 375.0 & 380.4 & 385.2 & 92 \\
\hline
\end{tabular}


Table :Toluene (1) - Cellosolve acetate (2) VLE by GE based models with regressed parameters at experimental P-T-x at P=61.32KPa

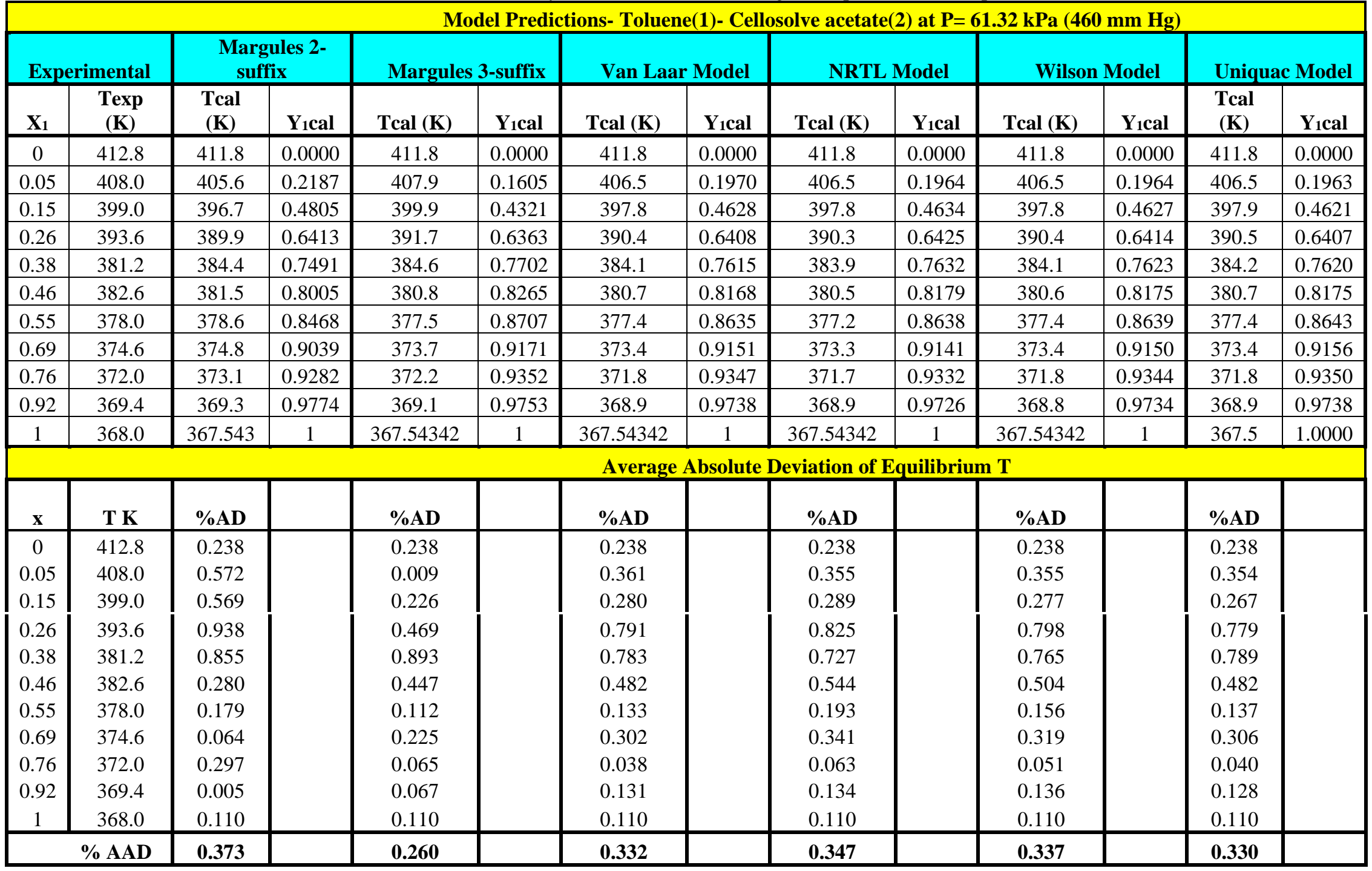


Table : Toluene (1) - Cellosolve acetate (2) VLE by GE based models with regressed parameters at experimental P-T-x at P=74.66 KPa.

\begin{tabular}{|c|c|c|c|c|c|c|c|c|c|c|c|c|c|}
\hline \multicolumn{14}{|c|}{ Model Predictions- Toluene(1)- Cellosolve acetate $(2)$ at $\mathrm{P}=74.66 \mathrm{kPa}(560 \mathrm{~mm} \mathrm{Hg})$} \\
\hline \multicolumn{2}{|c|}{ Experimental } & \multicolumn{2}{|c|}{$\begin{array}{l}\text { Margules 2- } \\
\text { suffix }\end{array}$} & \multicolumn{2}{|c|}{$\begin{array}{l}\text { Margules 3- } \\
\text { suffix }\end{array}$} & \multicolumn{2}{|c|}{ Van Laar Model } & \multicolumn{2}{|c|}{ NRTL Model } & \multicolumn{2}{|c|}{ Wilson Model } & \multicolumn{2}{|c|}{ Uniquac Model } \\
\hline $\mathbf{X}_{1}$ & $\begin{array}{c}T \exp \\
(\mathrm{K}) \\
\end{array}$ & $\begin{array}{c}\text { Tcal } \\
(\mathbf{K}) \\
\end{array}$ & Y 1 cal & $\begin{array}{c}\text { Tcal } \\
(\mathrm{K}) \\
\end{array}$ & Y,cal & Tcal (K) & Y 1 cal & Tcal (K) & Y 1 cal & Tcal (K) & Y 1 cal & Tcal $(\mathbf{K})$ & Y1cal \\
\hline 0 & 417.0 & 418.3 & 0.0000 & 418.3 & 0.0000 & 418.3 & 0.0000 & 418.3 & 0.0000 & 418.3 & 0.0000 & 418.3 & 0.0000 \\
\hline 0.05 & 412.4 & 412.1 & 0.2116 & 414.4 & 0.1552 & 412.9 & 0.1905 & 413.0 & 0.1893 & 413.0 & 0.1898 & 413.0 & 0.1902 \\
\hline 0.15 & 406.0 & 403.1 & 0.4682 & 406.3 & 0.4205 & 404.2 & 0.4507 & 404.3 & 0.4501 & 404.3 & 0.4503 & 404.3 & 0.4504 \\
\hline 0.26 & 398.0 & 396.2 & 0.6282 & 398.1 & 0.6235 & 396.8 & 0.6278 & 396.7 & 0.6283 & 396.8 & 0.6280 & 396.8 & 0.6280 \\
\hline 0.38 & 387.0 & 390.7 & 0.7372 & 390.9 & 0.7589 & 390.4 & 0.7499 & 390.3 & 0.7506 & 390.4 & 0.7504 & 390.4 & 0.7505 \\
\hline 0.46 & 388.4 & 387.7 & 0.7897 & 387.1 & 0.8167 & 387.0 & 0.8065 & 386.8 & 0.8069 & 386.9 & 0.8069 & 387.0 & 0.8073 \\
\hline 0.55 & 383.6 & 384.9 & 0.8375 & 383.8 & 0.8625 & 383.7 & 0.8549 & 383.5 & 0.8546 & 383.6 & 0.8551 & 383.6 & 0.8556 \\
\hline 0.69 & 378.4 & 381.0 & 0.8972 & 379.9 & 0.9111 & 379.6 & 0.9090 & 379.5 & 0.9077 & 379.6 & 0.9087 & 379.6 & 0.9093 \\
\hline 0.76 & 377.8 & 379.2 & 0.9229 & 378.4 & 0.9302 & 378.0 & 0.9297 & 377.9 & 0.9282 & 377.9 & 0.9293 & 378.0 & 0.9299 \\
\hline 0.92 & 375.0 & 375.5 & 0.9755 & 375.3 & 0.9733 & 375.0 & 0.9715 & 375.0 & 0.9708 & 375.0 & 0.9713 & 375.0 & 0.9716 \\
\hline 1 & 373.3 & 373.7 & 1 & 373.7 & 1 & 373.7 & 1 & 373.7 & 1 & 373.7 & 1 & 373.7 & 1.0000 \\
\hline \multicolumn{14}{|c|}{ Average Absolute Deviation of Equilibrium T } \\
\hline $\mathbf{x}$ & $\mathbf{T} \mathbf{K}$ & $\% \mathrm{AD}$ & & $\% \mathrm{AD}$ & & $\% \mathbf{A D}$ & & $\% \mathrm{AD}$ & & $\% \mathrm{AD}$ & & $\% \mathrm{AD}$ & \\
\hline 0 & 417.0 & 0.316 & & 0.316 & & 0.316 & & 0.316 & & 0.316 & & 0.316 & \\
\hline 0.05 & 412.4 & 0.069 & & 0.498 & & 0.144 & & 0.156 & & 0.151 & & 0.148 & \\
\hline 0.15 & 406.0 & 0.713 & & 0.089 & & 0.422 & & 0.414 & & 0.414 & & 0.414 & \\
\hline 0.26 & 398.0 & 0.444 & & 0.039 & & 0.292 & & 0.303 & & 0.293 & & 0.286 & \\
\hline 0.38 & 387.0 & 0.966 & & 1.017 & & 0.898 & & 0.870 & & 0.889 & & 0.899 & \\
\hline 0.46 & 388.4 & 0.159 & & 0.315 & & 0.357 & & 0.390 & & 0.370 & & 0.360 & \\
\hline 0.55 & 383.6 & 0.341 & & 0.057 & & 0.030 & & 0.001 & & 0.018 & & 0.025 & \\
\hline 0.69 & 378.4 & 0.699 & & 0.409 & & 0.329 & & 0.314 & & 0.321 & & 0.326 & \\
\hline 0.76 & 377.8 & 0.396 & & 0.163 & & 0.057 & & 0.052 & & 0.052 & & 0.058 & \\
\hline 0.92 & 375.0 & 0.147 & & 0.081 & & 0.015 & & 0.019 & & 0.014 & & 0.020 & \\
\hline 1 & 373.4 & 0.095 & & 0.095 & & 0.0948559 & & 0.095 & & 0.095 & & 0.095 & \\
\hline \multicolumn{2}{|c|}{$\%$ AAD } & 0.395 & & 0.280 & & 0.260 & & 0.266 & & 0.267 & & 0.268 & \\
\hline
\end{tabular}


Table : Toluene (1) - Cellosolve acetate (2) VLE by GE based models with regressed parameters at experimental P-T-x at P=87.99 KPa.

\begin{tabular}{|c|c|c|c|c|c|c|c|c|c|c|c|c|c|}
\hline \multicolumn{14}{|c|}{ Model Predictions- Toluene(1)- Cellosolve acetate $(2)$ at $\mathrm{P}=87.99 \mathrm{kPa}(660 \mathrm{~mm} \mathrm{Hg})$} \\
\hline \multicolumn{2}{|c|}{ Experimental } & \multicolumn{2}{|c|}{$\begin{array}{c}\begin{array}{c}\text { Margules 2- } \\
\text { suffix }\end{array} \\
\end{array}$} & \multicolumn{2}{|c|}{$\begin{array}{l}\text { Margules 3- } \\
\text { suffix }\end{array}$} & \multicolumn{2}{|c|}{ Van Laar Model } & \multicolumn{2}{|c|}{ NRTL Model } & \multicolumn{2}{|c|}{ Wilson Model } & \multicolumn{2}{|c|}{ Uniquac Model } \\
\hline $\mathbf{X}_{1}$ & $\begin{array}{c}\text { Texp } \\
(\mathbf{K})\end{array}$ & $\begin{array}{l}\text { Tcal } \\
(\mathbf{K})\end{array}$ & Yical & Tcal (K) & Yical & Tcal (K) & Y1cal & Tcal (K) & Yical & Tcal (K) & Yical & $\begin{array}{l}\text { Tcal } \\
(\mathbf{K})\end{array}$ & Yical \\
\hline 0 & 424.0 & 424.0 & 0.0000 & 424.0 & 0.0000 & 424.0 & 0.0000 & 424.0 & 0.0000 & 424.0 & 0.0000 & 424.0 & 0.0000 \\
\hline 0.05 & 418.2 & 417.7 & 0.2063 & 420.1 & 0.1512 & 418.6 & 0.1857 & 418.7 & 0.1839 & 418.6 & 0.1848 & 418.6 & 0.1856 \\
\hline 0.15 & 410.2 & 408.6 & 0.4586 & 412.0 & 0.4117 & 409.8 & 0.4415 & 409.9 & 0.4398 & 409.9 & 0.4407 & 409.8 & 0.4414 \\
\hline 0.26 & 402.2 & 401.7 & 0.6180 & 403.7 & 0.6135 & 402.3 & 0.6177 & 402.3 & 0.6171 & 402.3 & 0.6175 & 402.3 & 0.6180 \\
\hline 0.38 & 393.4 & 396.1 & 0.7277 & 396.4 & 0.7500 & 395.9 & 0.7407 & 395.9 & 0.7405 & 395.9 & 0.7408 & 395.9 & 0.7413 \\
\hline 0.46 & 393.4 & 393.2 & 0.7811 & 392.6 & 0.8088 & 392.4 & 0.7983 & 392.4 & 0.7980 & 392.4 & 0.7984 & 392.4 & 0.7990 \\
\hline 0.55 & 389.0 & 390.3 & 0.8301 & 389.2 & 0.8558 & 389.1 & 0.8480 & 389.0 & 0.8472 & 389.0 & 0.8479 & 389.0 & 0.8485 \\
\hline 0.69 & 383.2 & 386.4 & 0.8918 & 385.3 & 0.9062 & 384.9 & 0.9039 & 385.0 & 0.9025 & 384.9 & 0.9036 & 384.9 & 0.9041 \\
\hline 0.76 & 383.4 & 384.6 & 0.9185 & 383.7 & 0.9262 & 383.2 & 0.9256 & 383.3 & 0.9241 & 383.3 & 0.9252 & 383.3 & 0.9256 \\
\hline 0.92 & 380.4 & 380.8 & 0.9740 & 380.6 & 0.9715 & 380.3 & 0.9697 & 380.4 & 0.9692 & 380.3 & 0.9696 & 380.3 & 0.9697 \\
\hline 1 & 378.3 & 379.0 & 1 & 379.0 & 1 & 379.0 & 1 & 379.0 & 1 & 379.0 & 1 & 379.0 & 1.0000 \\
\hline \multicolumn{14}{|c|}{ Average Absolute Deviation of Equilibrium $T$} \\
\hline $\mathbf{x}$ & $\mathbf{T ~ K}$ & $\% \mathrm{AD}$ & & $\% \mathrm{AD}$ & & $\% \mathrm{AD}$ & & $\% \mathrm{AD}$ & & $\% \mathrm{AD}$ & & $\% \mathrm{AD}$ & \\
\hline 0 & 424.0 & 0.008 & & 0.008 & & 0.008 & & 0.008 & & 0.008 & & 0.008 & \\
\hline 0.05 & 418.2 & 0.105 & & 0.466 & & 0.110 & & 0.127 & & 0.118 & & 0.111 & \\
\hline 0.15 & 410.2 & 0.374 & & 0.439 & & 0.079 & & 0.057 & & 0.068 & & 0.076 & \\
\hline 0.26 & 402.2 & 0.117 & & 0.379 & & 0.039 & & 0.048 & & 0.044 & & 0.040 & \\
\hline 0.38 & 393.4 & 0.708 & & 0.769 & & 0.645 & & 0.640 & & 0.643 & & 0.642 & \\
\hline 0.46 & 393.4 & 0.049 & & 0.197 & & 0.245 & & 0.253 & & 0.249 & & 0.250 & \\
\hline 0.55 & 389.0 & 0.336 & & 0.057 & & 0.027 & & 0.021 & & 0.023 & & 0.022 & \\
\hline 0.69 & 383.2 & 0.839 & & 0.550 & & 0.467 & & 0.473 & & 0.467 & & 0.466 & \\
\hline 0.76 & 383.4 & 0.327 & & 0.094 & & 0.014 & & 0.001 & & 0.011 & & 0.011 & \\
\hline 0.92 & 380.4 & 0.130 & & 0.061 & & 0.007 & & 0.004 & & 0.005 & & 0.001 & \\
\hline 1 & 378.4 & 0.182 & & 0.182 & & 0.182 & & 0.182 & & 0.182 & & 0.182 & \\
\hline \multicolumn{2}{|c|}{$\% \mathrm{AAD}$} & 0.289 & & 0.291 & & 0.149 & & 0.165 & & 0.165 & & 0.164 & \\
\hline
\end{tabular}


Table : Toluene (1) - Cellosolve acetate (2) VLE by GE based models with regressed parameters at experimental P-T-x at P=101.325 KPa

\begin{tabular}{|c|c|c|c|c|c|c|c|c|c|c|c|c|c|}
\hline \multicolumn{14}{|c|}{ Model Predictions- Toluene(1)- Cellosolve acetate $(2)$ at $P=101.325 \mathrm{kPa}(760 \mathrm{~mm} \mathrm{Hg})$} \\
\hline \multicolumn{2}{|c|}{ Experimental } & \multicolumn{2}{|c|}{$\begin{array}{l}\text { Margules 2- } \\
\text { suffix }\end{array}$} & \multicolumn{2}{|c|}{$\begin{array}{l}\text { Margules 3- } \\
\text { suffix }\end{array}$} & \multicolumn{2}{|c|}{ Van Laar Model } & \multicolumn{2}{|c|}{ NRTL Model } & \multicolumn{2}{|c|}{ Wilson Model } & \multicolumn{2}{|c|}{ Uniquac Model } \\
\hline $\mathbf{X}_{1}$ & $\begin{array}{c}\text { Texp } \\
(\mathbf{K}) \\
\end{array}$ & $\begin{array}{l}\text { Tcal } \\
(\mathbf{K})\end{array}$ & Y1cal & $\begin{array}{l}\text { Tcal } \\
(\mathbf{K})\end{array}$ & Y1cal & Tcal $(\mathbf{K})$ & Y1cal & Tcal $(\mathbf{K})$ & Y 1 cal & Tcal $(\mathbf{K})$ & Y & $\begin{array}{l}\text { Tcal } \\
(\mathbf{K})\end{array}$ & Y1cal \\
\hline 0 & 429.2 & 429.1 & 0.0000 & 429.1 & 0.0000 & 429.1 & 0.0000 & 429.1 & 0.0000 & 429.1 & 0.0000 & 429.1 & 0.0000 \\
\hline 0.05 & 420.4 & 422.8 & 0.2021 & 425.2 & 0.1480 & 423.7 & 0.1819 & 423.8 & 0.1797 & 423.7 & 0.1809 & 423.7 & 0.1819 \\
\hline 0.15 & 415.4 & 413.6 & 0.4510 & 417.0 & 0.4047 & 414.8 & 0.4341 & 414.9 & 0.4316 & 414.9 & 0.4331 & 414.8 & 0.4342 \\
\hline 0.26 & 406.8 & 406.6 & 0.6097 & 408.6 & 0.6054 & 407.2 & 0.6095 & 407.3 & 0.6080 & 407.3 & 0.6091 & 407.2 & 0.6099 \\
\hline 0.38 & 400.0 & 401.0 & 0.7200 & 401.3 & 0.7426 & 400.7 & 0.7331 & 400.8 & 0.7322 & 400.8 & 0.7330 & 400.7 & 0.7338 \\
\hline 0.46 & 397.4 & 398.0 & 0.7741 & 397.4 & 0.8023 & 397.2 & 0.7916 & 397.3 & 0.7906 & 397.2 & 0.7914 & 397.2 & 0.7922 \\
\hline 0.55 & 393.6 & 395.0 & 0.8239 & 394.0 & 0.8503 & 393.8 & 0.8422 & 393.9 & 0.8410 & 393.8 & 0.8419 & 393.8 & 0.8427 \\
\hline 0.69 & 390.6 & 391.1 & 0.8872 & 390.0 & 0.9021 & 389.6 & 0.8997 & 389.8 & 0.8981 & 389.7 & 0.8992 & 389.7 & 0.8998 \\
\hline 0.76 & 388.4 & 389.4 & 0.9149 & 388.4 & 0.9228 & 388.0 & 0.9256 & 388.1 & 0.9206 & 388.1 & 0.9217 & 388.0 & 0.9221 \\
\hline 0.92 & 385.2 & 385.6 & 0.9726 & 385.3 & 0.9701 & 385.0 & 0.9697 & 385.1 & 0.9680 & 385.1 & 0.9681 & 385.1 & 0.9682 \\
\hline 1 & 384.15 & 383.7 & 1 & 383.7 & 1 & 383.7 & 1 & 383.7 & 1 & 383.7 & 1 & 383.8 & 1.0000 \\
\hline \multicolumn{14}{|c|}{ Average Absolute Deviation of Equilibrium T } \\
\hline 0 & 429.2 & 0.008 & & 0.008 & & 0.008 & & 0.008 & & 0.008 & & 0.008 & \\
\hline 0.05 & 420.4 & 0.574 & & 1.153 & & 0.792 & & 0.815 & & 0.802 & & 0.792 & \\
\hline 0.15 & 415.4 & 0.429 & & 0.393 & & 0.131 & & 0.097 & & 0.116 & & 0.131 & \\
\hline 0.26 & 406.8 & 0.045 & & 0.461 & & 0.114 & & 0.142 & & 0.125 & & 0.112 & \\
\hline 0.38 & 400.0 & 0.256 & & 0.325 & & 0.195 & & 0.212 & & 0.201 & & 0.190 & \\
\hline 0.46 & 397.4 & 0.156 & & 0.014 & & 0.039 & & 0.025 & & 0.035 & & 0.045 & \\
\hline 0.55 & 393.6 & 0.380 & & 0.106 & & 0.071 & & 0.087 & & 0.075 & & 0.066 & \\
\hline 0.69 & 390.6 & 0.147 & & 0.139 & & 0.225 & & 0.200 & & 0.216 & & 0.223 & \\
\hline 0.76 & 388.4 & 0.259 & & 0.024 & & 0.086 & & 0.057 & & 0.076 & & 0.080 & \\
\hline 0.92 & 385.2 & 0.110 & & 0.039 & & 0.031 & & 0.013 & & 0.026 & & 0.024 & \\
\hline 1 & 384.2 & 0.101 & & 0.101 & & 0.100 & & 0.101 & & 0.101 & & 0.101 & \\
\hline \multicolumn{2}{|c|}{$\%$ AAD } & 0.224 & & 0.251 & & 0.154 & & 0.160 & & 0.162 & & 0.161 & \\
\hline
\end{tabular}




\section{GE BASED EQUATION FOR BINARY MIXTURES:}

1. Margules 2 suffix model:

Model parameter: A

$$
\begin{array}{cc|}
P=X_{1} \gamma_{1} P_{1}^{\text {sat }}+X_{2} \gamma_{2} P_{2}^{\text {sat }} & \ln \gamma_{1}^{\infty}=\ln \gamma_{2}^{\infty}=\boldsymbol{A} \\
\boldsymbol{Y}_{\boldsymbol{i}}=\frac{\boldsymbol{X}_{\boldsymbol{i}} \gamma_{i} \boldsymbol{P}_{i}^{\text {sat }}}{\boldsymbol{P}} & \frac{\boldsymbol{G}^{\boldsymbol{E}}}{\boldsymbol{R} \boldsymbol{T} \boldsymbol{X}_{1} \boldsymbol{X}_{2}}=\boldsymbol{A} \\
\ln \gamma_{1}=A X_{2}^{2} & \ln \gamma_{2}=A X_{1}^{2}
\end{array}
$$

2. Margules 3 suffix model:

Model parameter: $\mathbf{A}_{\mathbf{1 2}}$ and $\mathbf{A}_{\mathbf{2 1}}$

$$
\begin{array}{ll}
P=X_{1} \gamma_{1} P_{1}^{\text {sat }}+X_{2} \gamma_{2} P_{2}^{\text {sat }} & \ln \gamma_{1}=X_{2}^{2}\left[A_{12}+2\left(A_{21}-A_{12}\right) X_{1}\right] \\
\boldsymbol{Y}_{\boldsymbol{i}}=\frac{\boldsymbol{X}_{\boldsymbol{i}} \gamma_{i} \boldsymbol{P}_{i}^{\text {sat }}}{\boldsymbol{P}} & \frac{G^{E}}{R T X_{1} X_{2}}=A_{21} X_{1}+A_{12} X_{2} \\
\ln \gamma_{2}=X_{1}^{2}\left[A_{21}+2\left(A_{12}-A_{21}\right) X_{2}\right] & \ln \gamma_{1}^{\infty}=A_{12} \quad \ln \gamma_{2}^{\infty}=A_{21}
\end{array}
$$


Table : Regressed Activity Coefficient Model Parameters.

\begin{tabular}{|c|c|c|}
\hline Model & Parameters & Best Value \\
\hline Margules 2-suffix Model & $\mathbf{A}$ & 0.41008394 \\
\hline \multirow[t]{2}{*}{ Margules 3-suffix Model } & A12 & -0.068947524 \\
\hline & $\mathbf{A 2 1}$ & 0.64200984 \\
\hline \multirow[t]{2}{*}{ Vanlaar Model } & A12 & 0.250650104 \\
\hline & A21 & 0.887837473 \\
\hline \multirow[t]{3}{*}{ NRTL Model } & b12 & -343 \\
\hline & b21 & 1089 \\
\hline & $\alpha$ & -2.961983475 \\
\hline \multirow[t]{2}{*}{ Wilson Model } & a12 & -675.233481 \\
\hline & a21 & 4047.48825 \\
\hline \multirow[t]{2}{*}{ Uniquac Model } & $\Delta \mathrm{u12}$ & 2160.261655 \\
\hline & $\Delta \mathrm{u} 21$ & -1284.739473 \\
\hline
\end{tabular}

Table : Final Result (\% AAD) at different pressure for $\mathrm{G}^{\mathrm{E}}$ Based Model

\begin{tabular}{|c|c|c|c|c|c|c|c|}
\hline \multicolumn{2}{|c|}{ Models } & $\begin{array}{l}\text { Margules 2- } \\
\text { suffix }\end{array}$ & Margules 3-suffix & Vanlaar & NRTL & Wilson & Uniquac \\
\hline $\mathbf{P}(\mathbf{k P a})$ & $\begin{array}{c}\mathrm{P}(\mathrm{mm} \\
\mathbf{H g})\end{array}$ & \multicolumn{6}{|c|}{$\Sigma(\Delta T)^{2}$} \\
\hline 61.3283 & 460 & 38.854 & 20.869 & 28.325 & 29.571 & 28.596 & 28.006 \\
\hline 74.6605 & 560 & 39.033 & 26.131 & 22.121 & 21.640 & 21.855 & 22.007 \\
\hline 87.9928 & 660 & 24.913 & 24.273 & 11.385 & 11.480 & 11.402 & 11.354 \\
\hline 101.3250 & 760 & 14.364 & 32.033 & 13.350 & 13.873 & 13.571 & 13.280 \\
\hline \multicolumn{2}{|c|}{ Average } & 29.291 & 25.826 & 18.795 & 19.141 & 18.856 & 18.662 \\
\hline $\mathbf{P}(\mathbf{k P a})$ & $\begin{array}{c}\mathrm{P}(\mathrm{mm} \\
\mathbf{H g})\end{array}$ & \multicolumn{6}{|c|}{ RMSD T } \\
\hline 61.3283 & 460 & 0.567 & 0.415 & 0.484 & 0.494 & 0.486 & 0.481 \\
\hline 74.6605 & 560 & 0.568 & 0.465 & 0.032 & 0.423 & 0.425 & 0.426 \\
\hline 87.9928 & 660 & 0.454 & 0.448 & 0.307 & 0.308 & 0.307 & 0.306 \\
\hline 101.3250 & 760 & 0.345 & 0.515 & 0.332 & 0.339 & 0.035 & 0.306 \\
\hline \multicolumn{2}{|c|}{ Average } & 0.483 & 0.461 & 0.289 & 0.391 & 0.313 & 0.380 \\
\hline $\mathbf{P}(\mathbf{k P a})$ & $\begin{array}{c}\mathbf{P}(\mathbf{m m} \\
\mathbf{H g})\end{array}$ & \multicolumn{6}{|c|}{$\%$ AAD T } \\
\hline 61.3283 & 460 & 0.373 & 0.260 & 0.332 & 0.347 & 0.337 & 0.330 \\
\hline 74.6605 & 560 & 0.395 & 0.280 & 0.269 & 0.423 & 0.267 & 0.268 \\
\hline 87.9928 & 660 & 0.289 & 0.291 & 0.166 & 0.165 & 0.165 & 0.164 \\
\hline 101.3250 & 760 & 0.224 & 0.251 & 0.163 & 0.339 & 0.153 & 0.161 \\
\hline \multicolumn{2}{|c|}{ Average } & 0.320 & 0.271 & 0.232 & 0.318 & 0.231 & 0.231 \\
\hline
\end{tabular}




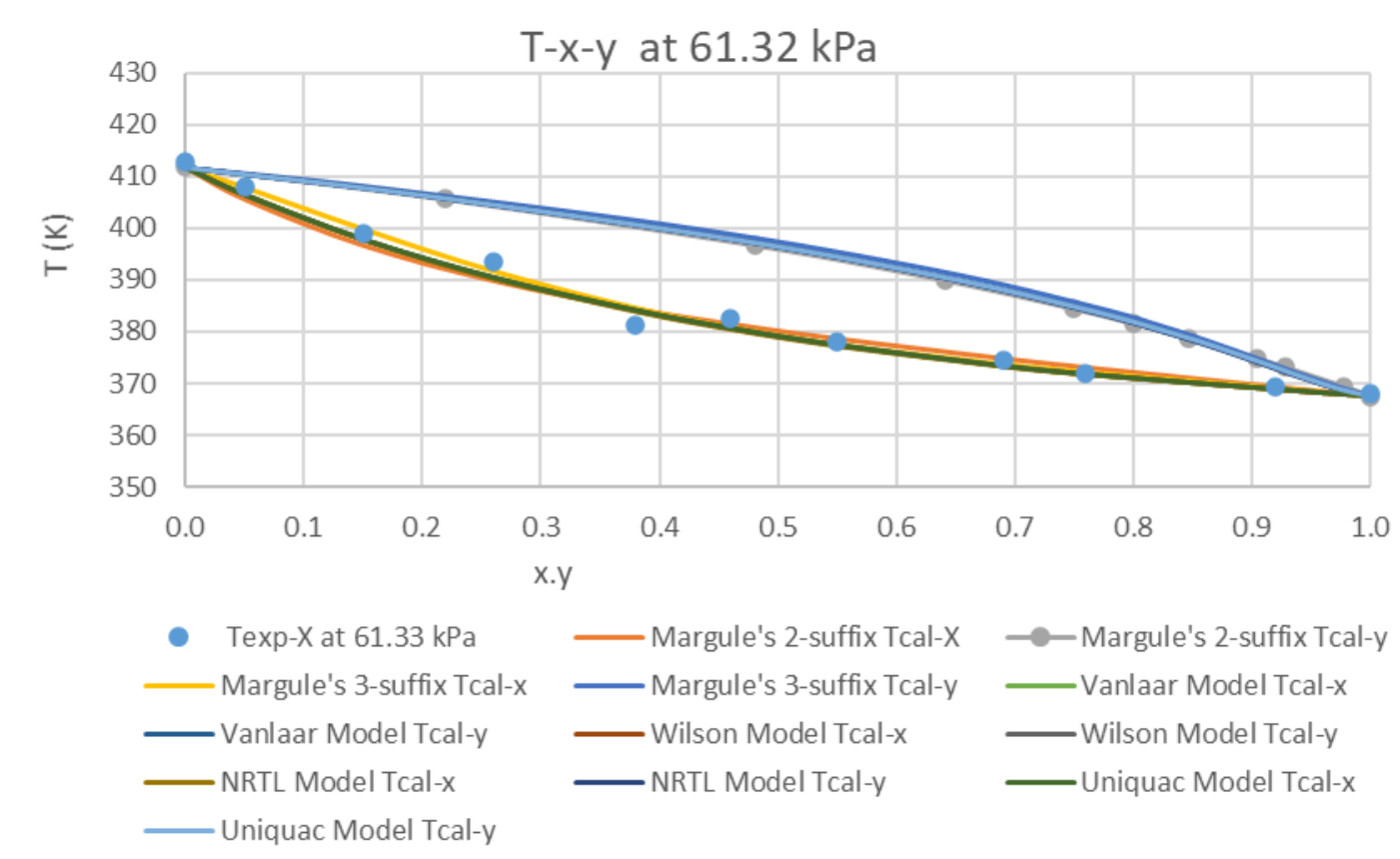

Figure : Toluene-Cellosolve Acetate $\mathrm{T}-\mathrm{x}-\mathrm{y}$ diagram at $\mathrm{P}=61.32 \mathrm{KPa}$.

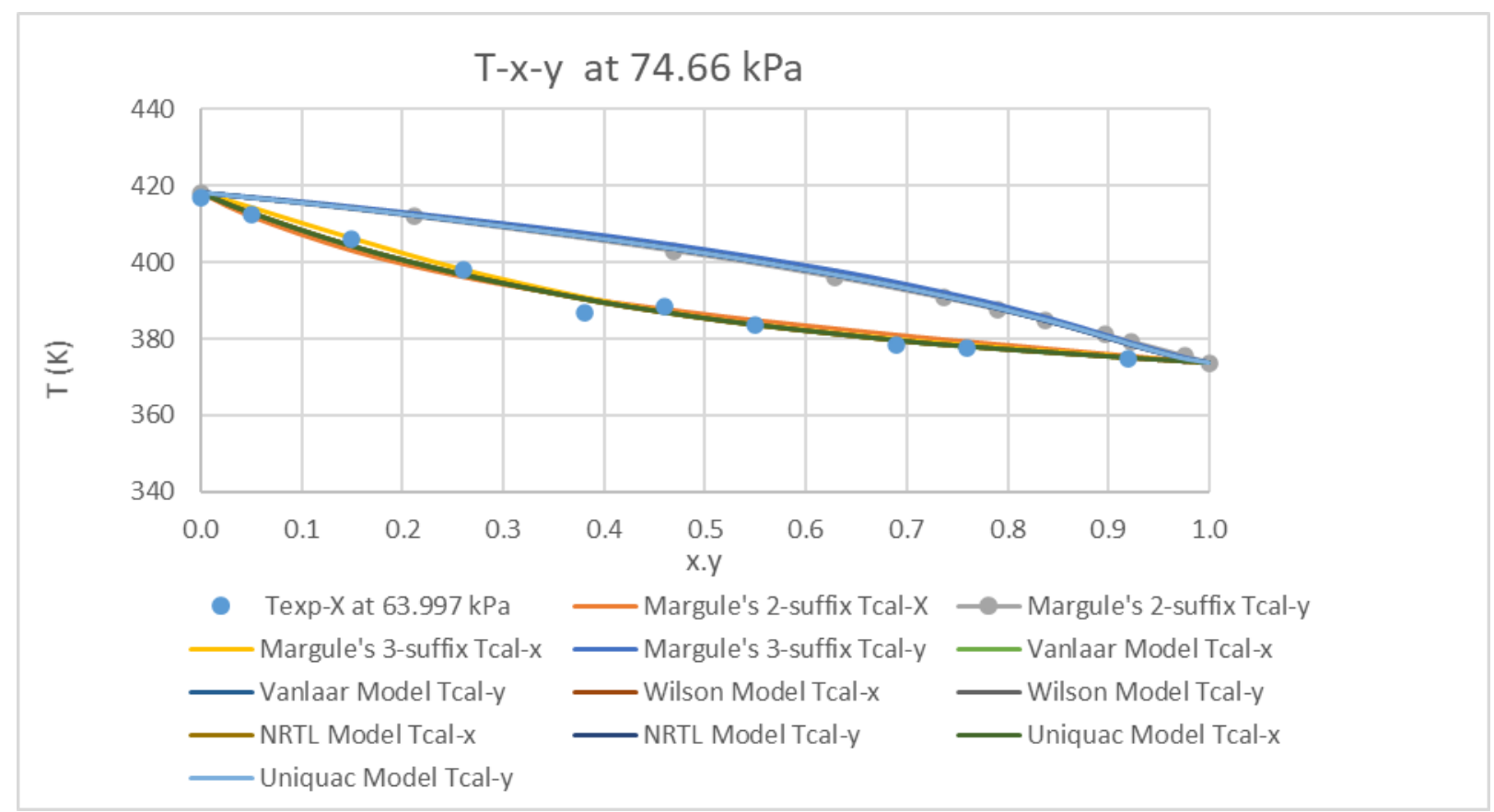

Figure : Toluene-Cellosolve Acetate T-x-y diagram at $\mathrm{P}=74.66 \mathrm{KPa}$. 


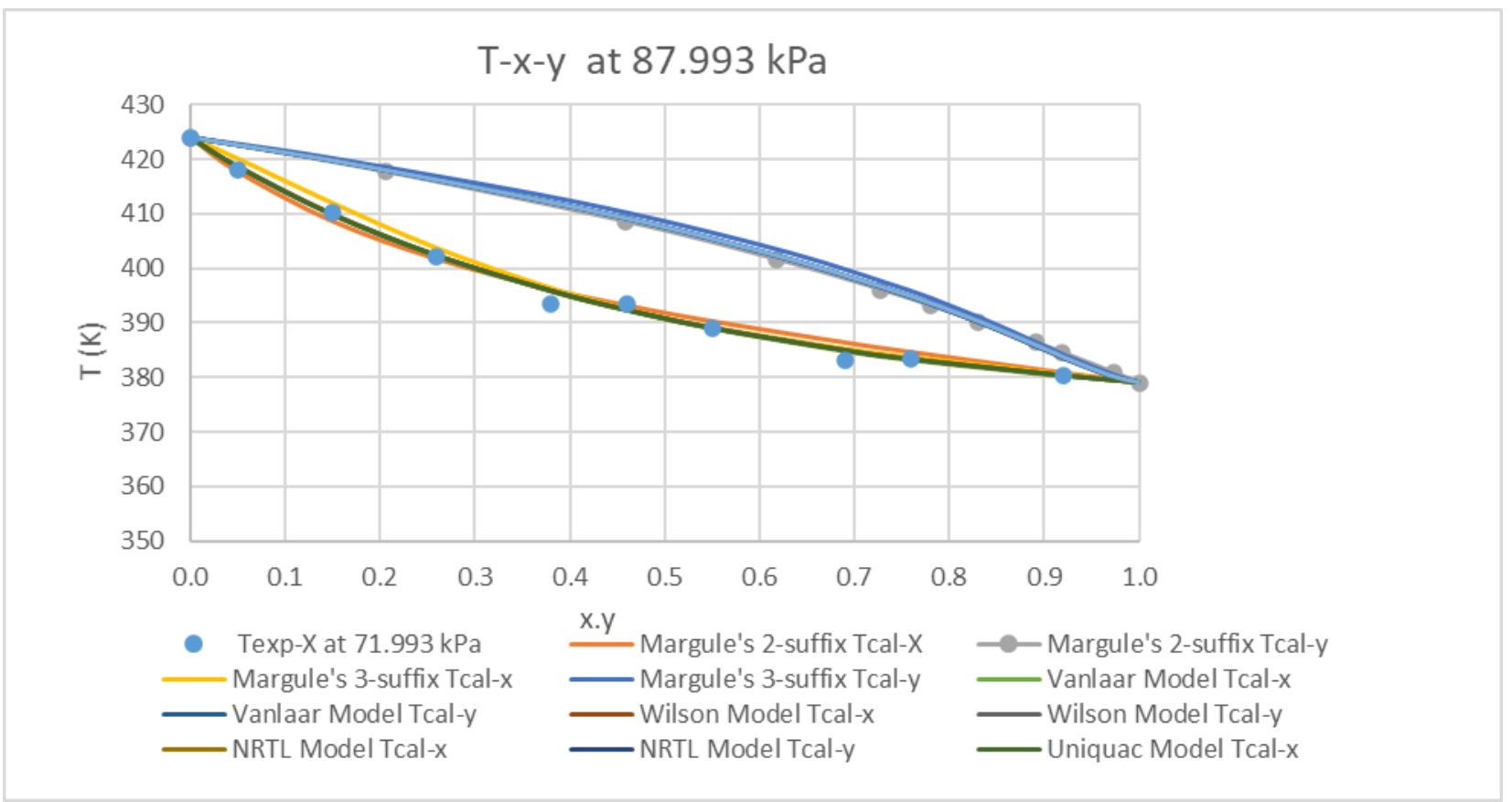

Figure : Toluene-Cellosolve Acetate T-x-y diagram at $\mathrm{P}=87.99 \mathrm{kPa}$.

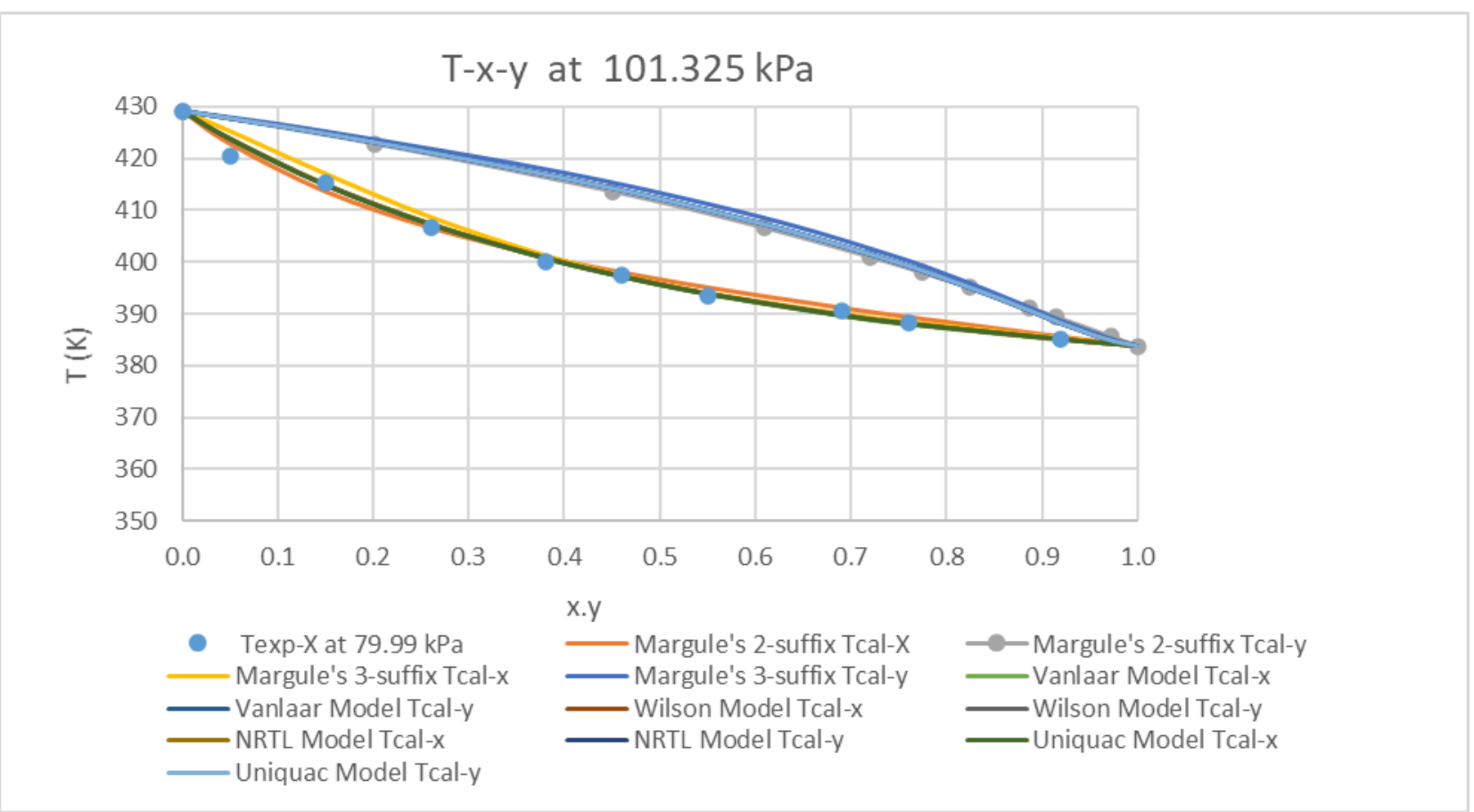

Figure : Toluene-Cellosolve Acetate T-x-y diagram at $\mathrm{P}=101.325 \mathrm{KPa}$ 


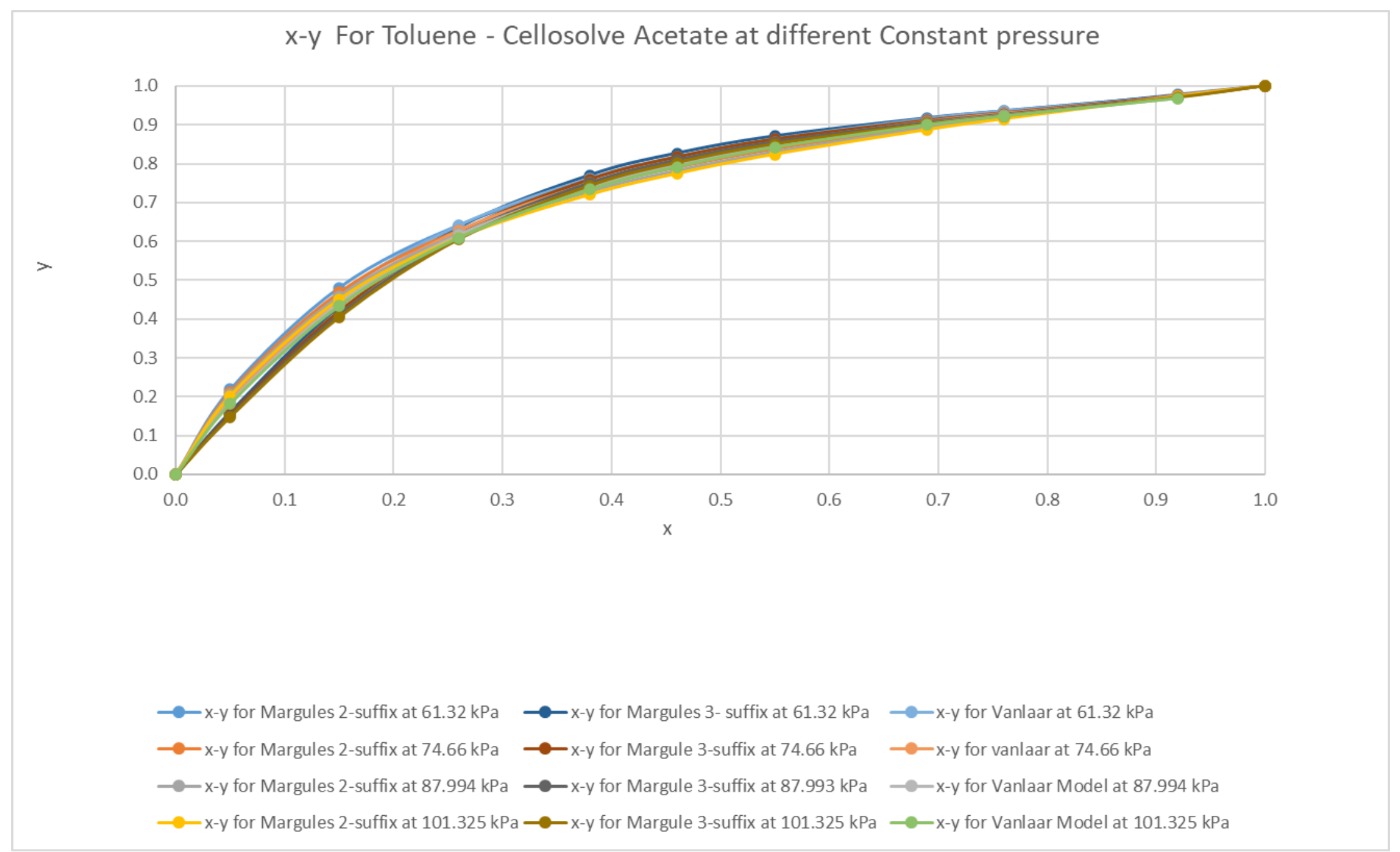

Figure : Toluene-Cellosolve Acetate $\mathrm{X}-\mathrm{Y}(\mathrm{VLE})$ by $\mathrm{G}^{\mathrm{E}}$ based models with regressed parameters at experimental P-T-x. 


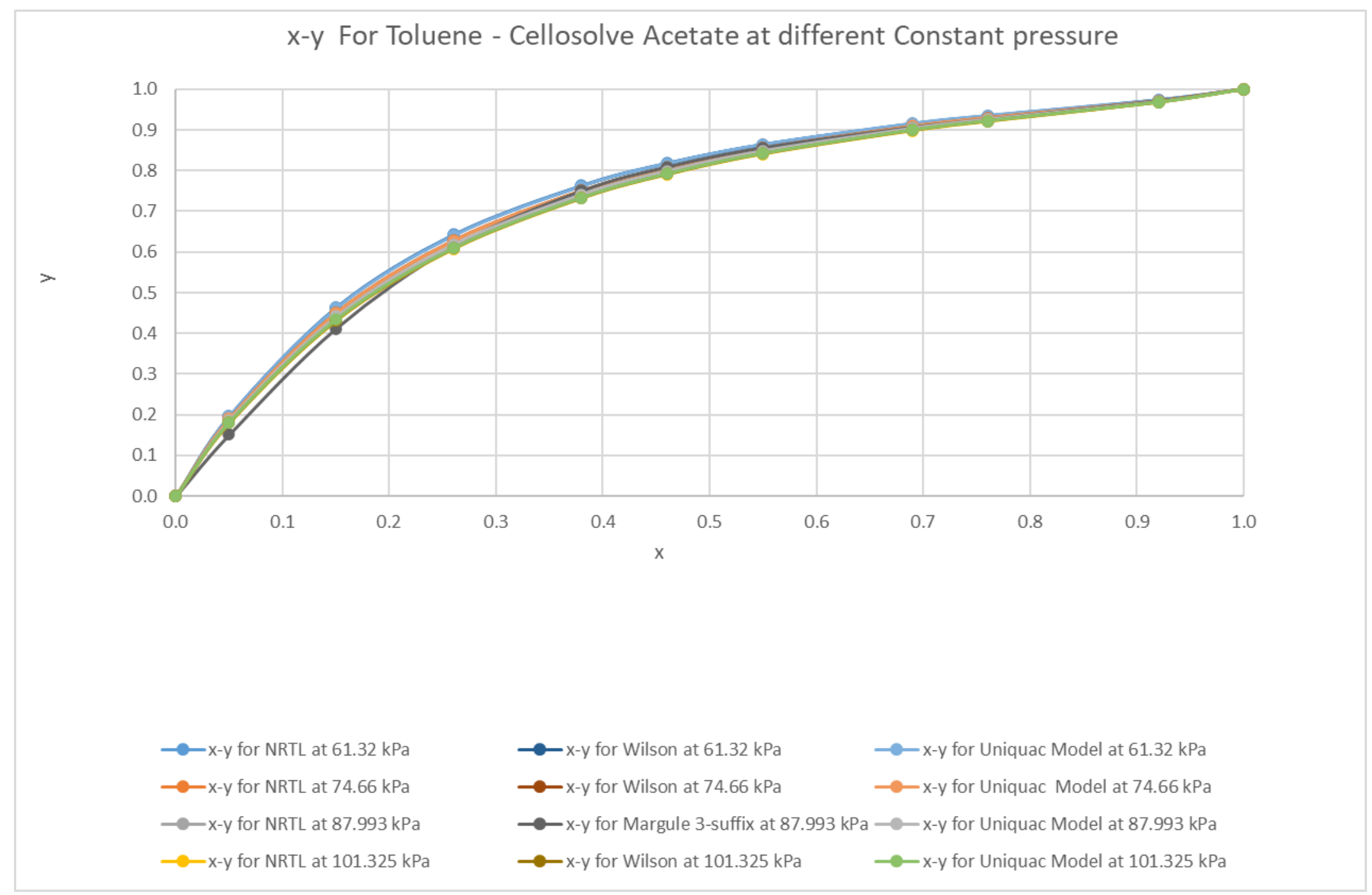

Figure : Toluene-Cellosolve Acetate $\mathrm{X}-\mathrm{Y}(\mathrm{VLE})$ by $\mathrm{G}^{\mathrm{E}}$ based models with regressed parameters at experimental P-T-x. 


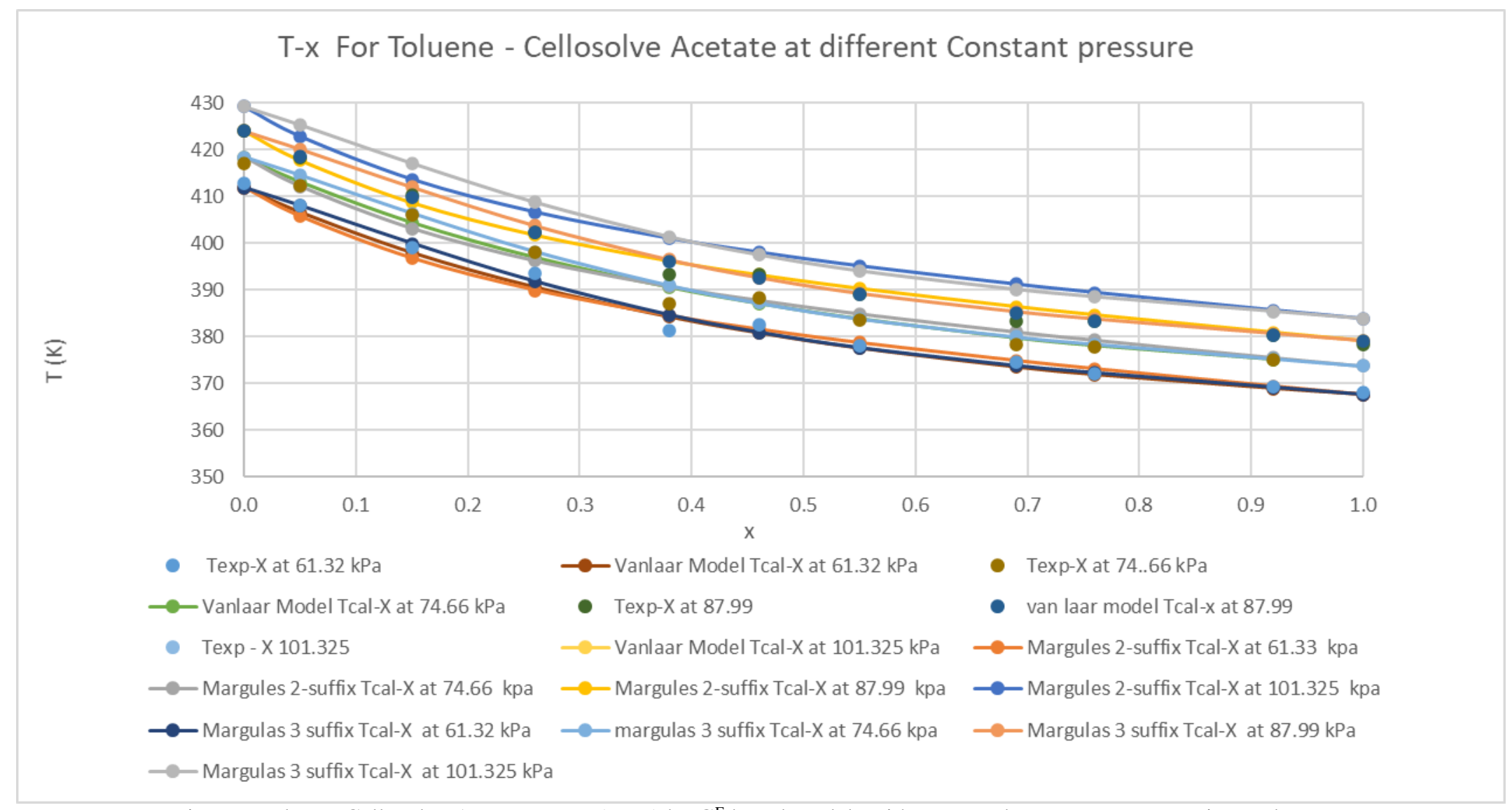

Figure : Toluene-Cellosolve Acetate $\mathrm{T}-\mathrm{X}(\mathrm{VLE})$ by $\mathrm{G}^{\mathrm{E}}$ based models with regressed parameters at experimental P-T-x. 


\section{CONCLUSION}

The experimental work in this project involved generating pure component and binary VLE data for Cellosolve Acetate (2-Ethoxyethyl Acetate) - Toluene. The purpose of taking up this work was that 2-ethoxyethyl acetate is synthesized by the esterification of 2-ethoxy ethanol and acetic acid using reactive distillation. To increase the yield of the product and minimize energy consumption toluene is added as an entrainer. This makes the knowledge of VLE of the species along with toluene also necessary. The data for the above-mentioned system was generated at four different pressures. The conclusions drawn from this study are given below:

1. Vapour pressure data for Toluene and Cellosolve Acetate were generated at four different pressures ranging $61.32 \mathrm{kPa}$ to $101.325 \mathrm{kPa}$. Antoine constants for both the species were regressed using excel.

2. Experimental, predicted and literature values of Vapour pressure for Toluene and Cellosolve acetate match.

\section{REFERENCES}

[1] Rana BK, Bhate NV, Mahajani SM, Dabke SP. Vapor-Liquid Equilibrium for the 2-Ethoxyethanol-2 - Ethoxyethyl Acetate System. J. Chem. Eng. Data. 2012, 57:3483-3487.

[2] Smith JM, Van ness HM, Abbott MM. Introduction to chemical engineering thermodynamics. The McGraw-Hill Companies, Inc. New York; 2003:346.

[3] Cortinovis GF, Salvagnini WM, Tavares DT, Taqueda ME. Estimation of Activity Coefficients for the Pairs of the System 2-Ethoxyethanol +2 -Ethoxyethyl Acetate +2 Butoxyethanol + 2-Butoxyethyl Acetate. J. Chem. Eng. Data. 2011, 56:4157-4163.

[4] Martin, MC, Cocero MJ, Mato RB. Vapour-liquid equilibrium data at $298.15 \mathrm{~K}$ for binary systems containing methyl acetate or methanol with 2-methoxyethanol or 2-ethoxyethanol. $J$. Chem. Eng. Data 1994, 39:535-537.

[5] Thomas EA, Newman BA, Nicolaides GL, Eckert CA. Limiting Activity Coefficients from Differential Ebulliometry. J. Chem. Eng. Data 1982, 27:233-240.

[6] Gorlova NN, Gredneva TM, Vasil'eva SA, Polyakova LV, Komarova LF. A study of liquid-vapor phase equilibrium in binary organic mixtures. Russ. J. Chem. 2001, 74 $1285-1288$. 